\title{
Colloidal Switches by Electric and Magnetic Fields
}

\section{Journal Article}

Author(s):

Demirörs, Ahmet F.; Beltramo, Peter J.; Vutukuri, Hanumantha Rao

Publication date:

2017-05-05

Permanent link:

https://doi.org/10.3929/ethz-b-000167906

Rights / license:

In Copyright - Non-Commercial Use Permitted

Originally published in:

ACS Applied Materials \& Interfaces 9(20), https://doi.org/10.1021/acsami.7b02619

Funding acknowledgement:

148040 - Bioinspired Composites by External Fields (SNF) 


\title{
Colloidal Switches by Electric and Magnetic
} Fields

\author{
Ahmet Faik Demirörs ${ }^{* a}$, Peter Beltramo ${ }^{b}$ and Hanumantha Rao Vutukuri ${ }^{b}$ \\ ${ }^{a}$ Complex Materials, Department of Materials, ETH Zurich, 8093 Zurich, Switzerland \\ ${ }^{b}$ Soft Materials, Department of Materials, ETH Zurich, 8093 Zurich, Switzerland \\ E-mail: ahmet.demiroers@mat.ethz.ch \\ Phone: +41446326431
}

Keywords: colloids, optical switch, directed self-assembly, electric fields, magnetic fields

\begin{abstract}
External electric and magnetic fields have already been proven to be a versatile tool to control particle assembly, however, the degree of control of the dynamics and versatility of produced structures is expected to increase if both can be implemented simultaneously. For example, while micromagnets can rapidly assemble superparamagnetic particles, repeated, rapid, disassembly or reassembly is not trivial due to remanence and coercivity of metals used in such applications. Here, an interdigitated design of micromagnet and micro-fabricated electrode enables rapid switching of colloids between the magnetic and electric potential minima. Active control over colloids between two such adjacent potential minima enables a fast on/off mechanism, which is potentially important for optical switches or display technologies. Moreover, we demonstrate that the response time of the colloids between the states is on the order of tens of milli seconds, which is tunable by electric field strength. By carefully designing the electrode pattern, our strategy enables us to switch the assembly of single particles down to few microns and also hierarchical assemblies containing many particles. Our work on precise dynamic control over
\end{abstract}


the particle position would open new avenues to find potential applications in optical switches and display technologies.

\section{Introduction}

Assembly of colloidal particles into various types of regular arrays/lattices is one of the fundamental challenges of colloid science motivated by the applications of such structures in photonics ${ }^{1-3}$, phononics $^{4,5}$, electronics ${ }^{6}$, sensors ${ }^{7,8}$. Since the pioneering works of Yablonovitch et al ${ }^{9,10}$ and van Blaaderen et $\mathrm{al}^{11}$, a wide range of strategies have been developed to prepare colloidal lattices and assemblies. Examples include drying/dewetting of the colloidal solutions ${ }^{12,13}$, templated assembly on micropatterned supports ${ }^{14-16}$, use of varous interparticle forces (e.g., electrostatic ${ }^{16-18}$, van der Waals $^{19}$, capillary ${ }^{20}$, magnetic ${ }^{21-23}$ ), as well as assembly schemes based on particle size/shape variety $^{24,25}$ and chemical patchiness ${ }^{26}$. Although existing assembly schemes can flexibly position colloids into desired structures (from crystalline lattices ${ }^{27,28}$, through open-lattice arrangements ${ }^{29}$, to arrays of well-defined colloidal clusters ${ }^{15,30}$ ), dynamic control of the position and symmetry/structure of the assembly reversibly remains challenging. In recent demonstrations of assembly/disassembly of colloids $^{21,31-33}$, only one type of external field was applied, which lacked control over the structure when the field is off. Such systems depend solely on the random thermal forces for the disassembly part, which typically slows the response time of the system for applications. For example, the Brownian time scale for a micrometer scale particle to diffuse over its radius is $\sim 2 \mathrm{~s}$, considering that the Brownian time scale of diffusion can be written as $\tau=a^{2} / 4 D$ where $a$ is the particle radius and $D$ is the diffusion coefficient.

Having two potential minima adjacent to each-other and being able to rapidly switch between these two assembly states is a powerful tool for optics, especially in obtaining polarization-independent optical switches. Examples of possible uses of such optical switches are spatial light modulators, optical attenuators, and shutters ${ }^{34,35}$. Recently, polymer-dispersed liquid crystals (PDLC) ${ }^{36}$ or electrowetting systems ${ }^{37}$ were introduced as possible optical switches. Although PDLC is simple to fabricate, it had a forward scattering problem which made it sensitive for attenuation applications ${ }^{34,35}$. 
An electrowetting device ${ }^{37}$ uses two immiscible liquids: one is an opaque liquid (oil) spread over the device, while second liquid, i.e. water covers the oil. In the default case without any electric field the device stays opaque due to the oil, however, when a sufficiently high electric field is applied the oil film elongates along the field and forms a droplet instead of being spread over the device. Scaling electrowetting devices is challenging because increasing the area of the device increases the response time, which depends on the shrinking time of the oil. Dielectrophoresis was also used recently for optical switching and for light modulation by using shape changes of polydisperse macroscopic droplets upon application of an external field. These droplets, embedded in a sample cell, were covered by an opaque oil and elongation of the oil droplet opened transparent channels throught the device ${ }^{35}$ where the polydispersity of the droplets and lack of control in the droplet size limited the efficiency of fabrication. Dielectrophoretic devices had complications of miniaturization due to requirements on fine tuning the ratio of the thickness of droplet to the height of the device. Due to these limitations, the realization of novel optical switch mechanisms with enhanced response times, easy miniaturized fabrication schemes, and with superior spatial resolution still remains a major challenge.

Here, we present a novel self-assembly method using a combination of electric and magnetic fields and combine these two assembly techniques on a single chip, with as low as $5 \mu \mathrm{m}$ features. This strategy allows to switch between the potential minima promoted by magnetic and electric fields. Immediate response of the particles to the external fields exhibits an optical switch behaviour as the transparency/fluoresecence of the sample significantly changes, which is a direct consequence of the switch between magnetophoretic (MAG) and the dielectrophoretic (DEP) equilibrium states. Additionally, our method enables easy miniaturization, as the particles are monodisperse and micronsized and the electrode features we used were down to $5 \mu \mathrm{m}$. In addition, while one switching unit can be as small as few microns, the whole device formed from an array of such repeating units can be fabricated in sizes as large as a wafer. Furthermore, our method opens a novel way to actively control the assembly of colloidal particles and reassemble them, which is promising for microactuation research. Colloids in an electric field gradient experience forces due to the field gradient and the dielectric constant contrast between the medium and the particle. The dielectrophoretic 
force experienced by the particles is given by the following formula:

$$
F_{\mathrm{DEP}}=2 \pi r^{3} \varepsilon_{m} \operatorname{Re}\left\{\frac{\varepsilon_{p}^{*}-\varepsilon_{m}^{*}}{\varepsilon_{p}^{*}+2 \varepsilon_{m}^{*}}\right\} \nabla\left|\vec{E}_{r m s}\right|^{2}
$$

where $\varepsilon_{m}$ is the dielectric constant of the medium, $\varepsilon_{p}$ is the dielectric constant of the suspended particle, $r$ is the radius of the particle, and the factor in brackets is known as the complex ClausiusMossotti function ${ }^{38,39}$ which contains the information about frequency dependence of the DEP forces.

Similarly, particles in a magnetic field gradient experience forces depending on the magnetic field gradient and the contrast in magnetic susceptibilities of the medium and the particle. For instance, superparamagetic particles, suspended in a diamagnetic medium (e.g. water), provide quite a significant contrast in magnetic susceptibility, which forms the ground for magnetic manipulation of such particles. The magnetophoretic force experienced by the suspended particles is given by the following formula:

$$
F_{\mathrm{MAG}}=2 \pi r^{3} \chi_{m} \operatorname{Re}\left\{\frac{\chi_{p}^{*}-\chi_{m}^{*}}{\chi_{p}^{*}+2 \chi_{m}^{*}}\right\} \nabla|\vec{H}|^{2}
$$

where $\chi_{m}$ is the magnetic susceptibility of the medium and $\chi_{p}$ is the magnetic susceptibility of the suspended particle ${ }^{40}$. In both magnetophoresis and dielectrophoresis, forces that particles experience are directly related to the magnetic susceptibility and the dielectric permeability contrast between the particle and the medium, respectively. Using a superparamagnetic particle provides the susceptibility contrast $\left(\chi_{p}>\chi_{m}\right)$ for magnetic manipulation of the particles. Our superparamagnetic particle was a composite of iron oxide and polystyrene, which roughly resulted a dielectric constant of about 4.5, for $20 \mathrm{w} \%$ of iron oxide, estimated by using rule of mixtures. When suspended in a solvent like water $(\varepsilon=80.4)$ or DMSO $(\varepsilon=47)$ superparamagnetic particles will have a significant dielectric permeability contrast with the medium, which allows for manipulation by both magnetic and electric fields.

\section{Results and Discussion}

Recently, we developed an elegant method to control particle and cluster assembly using magnetic moulds via microfabricated ferromagnetic metal microdomains that acted as micromagnets when 

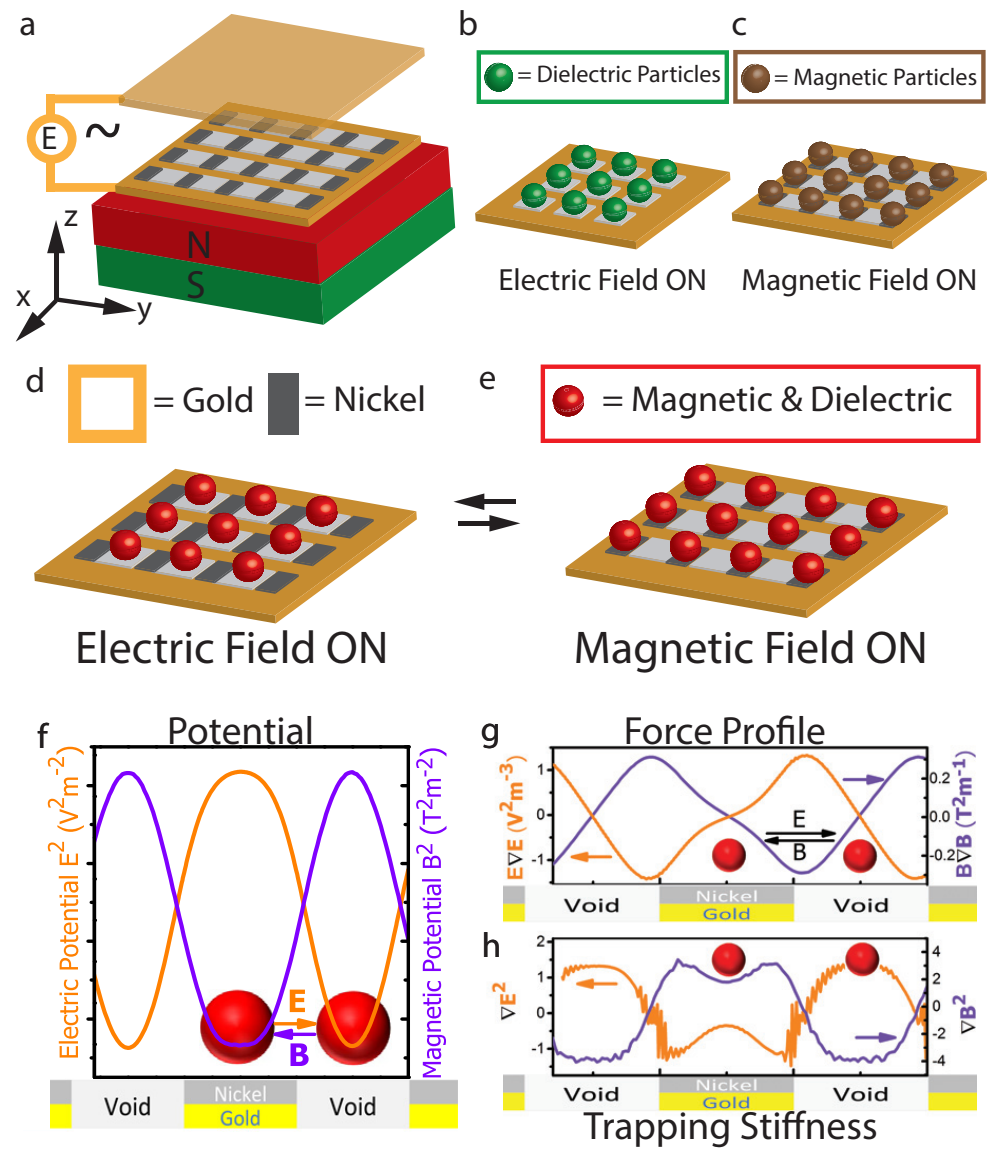

Figure 1: Electrode modeling and design. Final experimental setup with microfabricated Au and $\mathrm{Ni}$ patches (a) with the expected behavior of a dielectric (b) or magnetic (c) particle. A particle with both magnetic susceptibility and dielectric contrast is expected to switch between the electric (d) and magnetic (e) potential minima. Calculations of the magnetophoretic (purple) and dielectrophoretic (orange) potentials (f), force profiles (g) and trapping stiffness (h) for the particles used in experiments. Equilibrium particle locations occur at positions of force equal/close to zero in the force profiles and at the local maxima in the trapping stiffness calculations.

magnetized by a permanent magnet ${ }^{23}$. However, reversible disassembly of these assembled structures was not straightforward. High remanence and coercivity by the iron oxide (within the colloid) and nickel (patches on the electrode) caused the particles to remain magnetized after the cessation of the magnetic field. Thus, on-demand disassembly of superparamagnetic particles from their magnetic equilibrium states can not be achieved by thermal fluctuations. In order to push the superparamagnetic particles from their localization at high magnetic field regions, we expect dielectrophoretic forces to prove useful by driving them away from their magnetic-potential minimum and assemble them at another localization. By carefully designing two adjacent potential landscapes, using in- 
dependently controlled electric and magnetic fields, it is possible to switch the potential landscape such that particles start moving from their magnetostatic equilibrium positions to dielectrophoretic equilibrium positions or vice versa.

Design of such a chip with a micropatterned electrode and micromagnets, which enabled a potential minimum in the vicinity of the minimum of the other potential, is shown in Figure 1a-e. Figure 1a shows the experimental setup used to apply electric and magnetic fields, with a microfabricated bottom electrode with gold and nickel features. Figure 1b shows electric field directed-assembly of dielectric particles over a micropatterned electrode (array of square micro-voids on Au film) where particles have a lower dielectric constant compared to the medium $\left(\varepsilon_{p}<\varepsilon_{m}\right)$. Figure 1c shows the design of microfabricated electrodes with ferromagnetic nickel patches and the expected assembly of superparamagnetic particles over the nickel domains, when a magnetic field is applied. When superparamagnetic particles with desired dielectric properties $\left(\varepsilon_{p}<\varepsilon_{m}\right.$, red magnetodielectric particles in Figure 1d-e) are used, position of the particles can be changed from voids (electric) to nickel patches (magnetic) by applying electric or magnetic fields, respectively. Numerical calculations were performed in order to estimate the potential and force field profiles acting on particles in space (Supplementary Information Figure S1). The potential and forces acting on colloidal particles (with both superparamagnetic and dielectric properties) due to the applied fields are shown in Figure $1 \mathrm{f}$ and $1 \mathrm{~g}$, respectively. When electric field is applied (orange), particles are pushed towards the voids and in case of a magnetic field (purple) particles will be driven towards the nickel patches. The equilibrium positions of the particles with respect to the applied field is better appreciated with the trapping stiffness calculations, shown in Figure 1h. The maxima for the purple and orange plots, respectively, depict the most likely positions of particles, when a magnetic or an electric field is applied.

Sample cells, based on these calculations were fabricated by standard photolithography and involved the following steps, dissolution of the exposed photoresist regions, e-beam evaporation of the 40-nm gold layer, and lift-off of the masking photoresist by sonication in acetone. In a second round a $200 \mathrm{~nm}$ nickel layer was deposited similarly over specific sections of the gold electrode. When sample cells were filled with the superparamagnetic particles (4.69 $\mu \mathrm{m}$ size (microparticles $\mathrm{GmbH}$, 


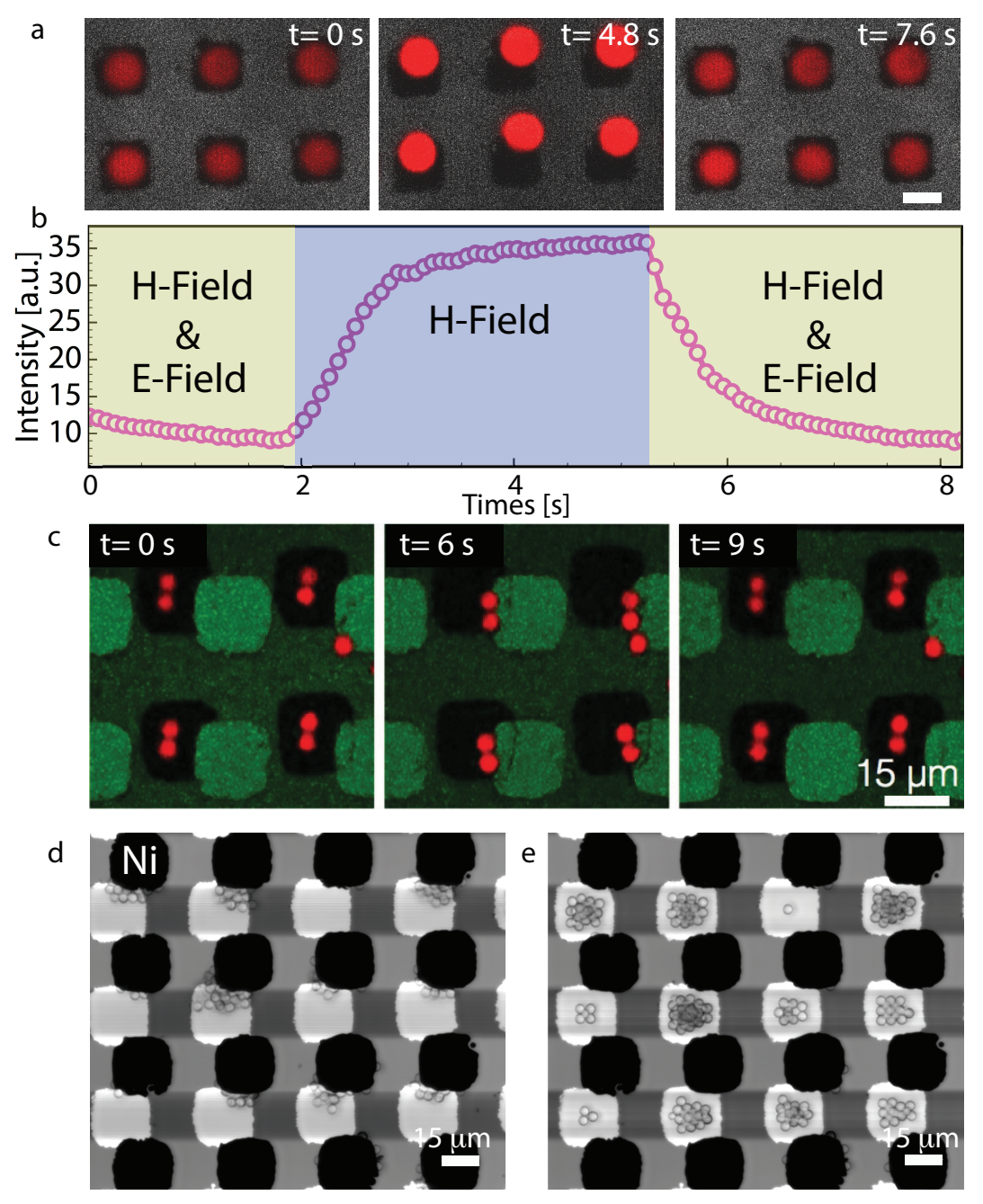

Figure 2: Dynamic switching of magneto-dielectric polystyrene particles between the two potential minima using magnetic and electric fields. a) Time-lapse fluorescent micrographs show the reversible switching of the position of $4.69 \mu \mathrm{m}$ particles between the two potential minima. b) Difference in fluorescence intensity reveals the positional change between the two states. c) Time-lapse fluorescent micrographs for the reversible switching of two $4.69 \mu \mathrm{m}$ particles between the two potential minima. d-e) Reflection mode images show the dynamic switching of the position of smaller particles (2.85 $\mu \mathrm{m}$ ) when the magnetic field is applied (d) and the electric field is applied (e). Note that, reflection images clearly show the positions of the nickel domains over the gold patterned electrode, see Figures S2 and S3. Scale bar in (a) is $5 \mu \mathrm{m}$.

PS-MAG-RhB-S2538) and $2.85 \mu \mathrm{m}$ size (Bangs Laboratories, Inc., UMC3F), a magnetic field of 2-3 $\mathrm{mT}$ was enough to bring the particles over the nickel domains. We applied an AC electric field of maximum $0.25 \mathrm{~V} / \mu \mathrm{m}$ (with a frequency of $1 \mathrm{MHz}$ to avoid electrohydrodynamic effects) to bring the particles over the voids of the microfabricated electrode. Panels of Figure 2a demonstrates how the position of the $4.69 \mu \mathrm{m}$ sized colloids, changes when an electric field is turned on and off while a 
magnetic field is continuously applied. Applying an electric field switches the location of the colloids back to the voids of the electrode pattern. Figure $2 \mathrm{~b}$ shows that the fluorescence intensity increases when the electric field is turned off and the magnetic field directs particles towards the Ni patches. The Ni patches are $200 \mathrm{~nm}$ higher than the outer gold electrode, and this change in height causes the particles to move out of the focal plane when the electric field is turned on. The height difference between the Ni patch and the void causes an additional gravitational potential energy barrier (order $\sim k \mathrm{~T})$, however, this is insignificant comparent to the electric and magnetic field strength $\left(\sim 10^{4}\right.$ $k \mathrm{~T})$. The spatial switch of the colloid from its magnetic potential minima to its electric potential minima occurs when an electric field is applied. However, this motion of the colloid is not only a lateral motion $(x$ and $y$ ) but has also a vertical $(z)$ component. This vertical motion is caused by the height difference of the void of the gold grid and the nickel patch on the grid. We measured the fluorescence intensity of the particles between two minima while we kept the Z-position (focal plane) fixed. Therefore, the fluorescence intensity count decreases as the particle moves out of focus. By exploiting the fluorescence intensity difference between these two potential minima, we were able to characterize the dynamics of colloidal switching together with tracking the position of the particle via image analyses (see Figure 3). To illustrate the flexibility of the approach to also reversibly assemble multiple particles, Figure 2c shows the assembly of two $4.69 \mu \mathrm{m}$ particles on a larger electrode patch $(20 \mu \mathrm{m})$ and 2-e show the assembly of $2.85 \mu \mathrm{m}$ particles on such an electrode $(20 \mu \mathrm{m})$ for multiple particles. By choosing appropriate void sizes, particle sizes, and suspension concentrations, we can in principle control the number of particles within each patch and dynamically assemble particle clusters. Figures $2 \mathrm{~d}$ and $2 \mathrm{e}$ show a similar realization of the switching of the assembly through different external fields for the $2.85 \mu \mathrm{m}$ superparamagnetic particles, imaged in reflection mode. Assemblies of several particles changed their location reversibly when the applied external field was switched. The nickel patches are better recognized in these images and in addition, when a magnetic field is applied, most particles accumulate on the edges of these islands, which is due to the magnetic field gradient over the nickel island and was reported before ${ }^{23}$. The movies of the switch of the assembly in time due to external-field changes are given in Supplementary Movies S1 and S2. 

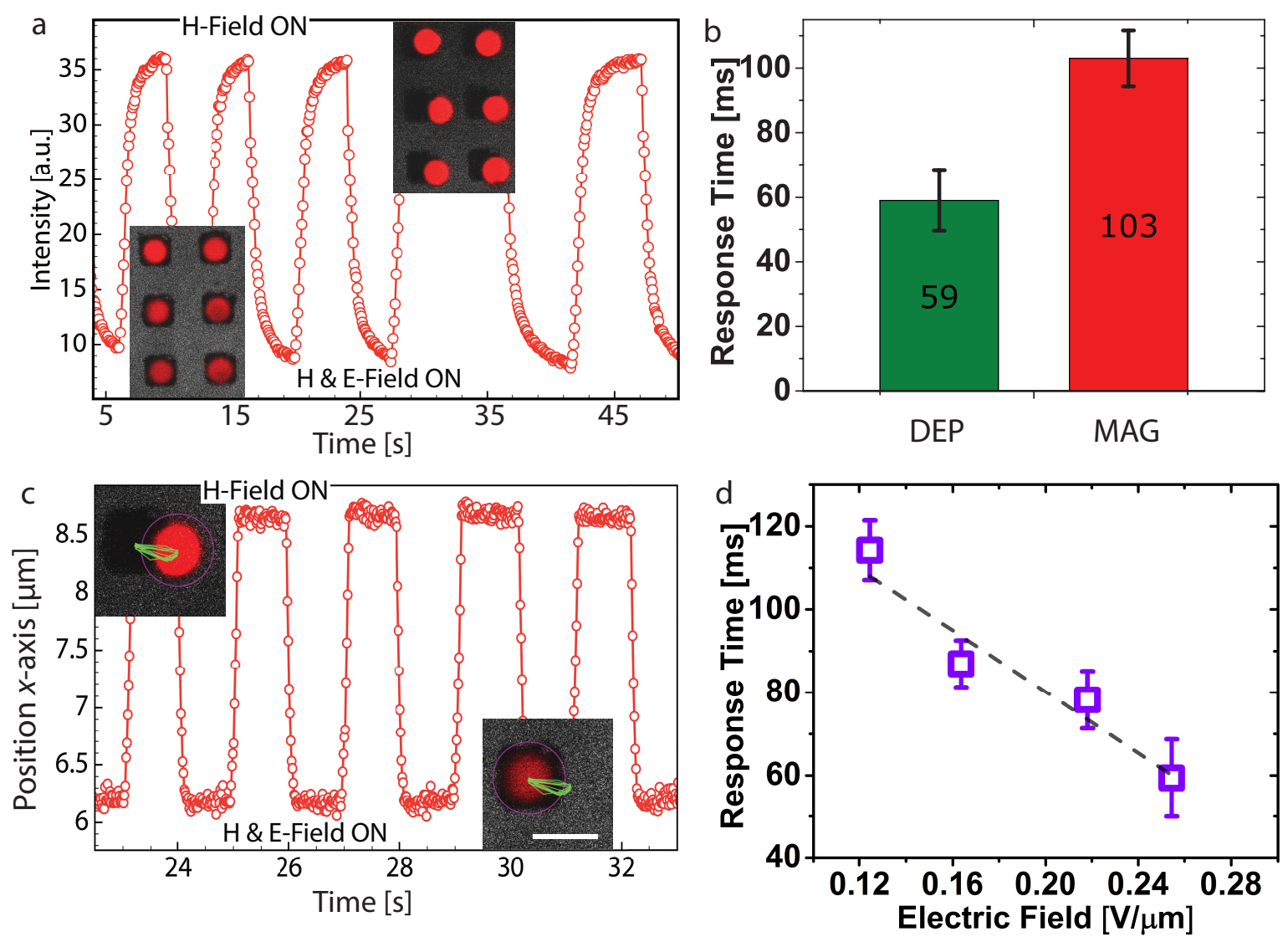

Figure 3: Characterization of the particles $(4.69 \mu \mathrm{m})$ response between the two potential minima for different field strengths. a) Active control over the position of particles as indicated by the sequential change in the fluorescence intensity of voids. b) The average response time of the particles between the two minima. c) Dynamic switching between the two minima as indicated by the positional change of the center of mass of a particle. Green line represents the trajectory of the centroid of the particle overlaid on a fluorescent image. Note that we kept the magnetic field is constant through out the experiment and we varied the electric field strength. d) Experimental response time with DEP switching is plotted as a function of applied field, which is changing linearly. Scale bar in (c) is $5 \mu \mathrm{m}$.

Dynamic switching of the colloidal assembly can find potential applications such as an optical switch, optical shutter or optical attenuator. Recently reported optical switches had response times down to $10 \mathrm{~ms}$ (i.e. $18 \mathrm{~ms}$ and $32 \mathrm{~ms}$ for rise and decay by Ren et al. ${ }^{35}$ and $10 \mathrm{~ms}$ for both rise and decay by Hayes et $a l .{ }^{37}$ ). In order to characterize the response time of our system, we followed the trajectories of particles over the two states while switching the external fields in time. By comparing the fluorescence intensity of the area over time as shown in Figure 3a, we addressed the reversibility of the system as well as the response time of the switching. Figure 3 clearly demonstrates the 
reversible modulation of the fluorescence intensity due to the switching of the fields. We found that the response time for switching from high to low intensity (due to electric field application) is faster than switching from low to high intensity (due to magnetic field application). This actually is a direct reflection of the strength of the external fields that takes part during the process. We apply a magnetic field of 2-3 mT, whereas the maximum electric field, we applied, was $0.25 \mathrm{~V} / \mu \mathrm{m}$. At these conditions, the electric response time of the particles was $\sim 59 \mathrm{msec}$, while the magnetic response time of the particles was $\sim 103 \mathrm{msec}$ as shown in Figure 3b. The measured response times are in quantitative agreement with the applied DEP and MAG forces. For instance, typical DEP forces we have in our system for a $0.1-0.25 \mathrm{~V} / \mu \mathrm{m}$ electric field strength is around $2-10 \mathrm{pN}$ and if we consider a constant acceleration of the colloid of $5 \mu \mathrm{m}$ diameter and assume that the colloid will move one diameter away, i.e. $\triangle x=5 \mu \mathrm{m}$, we can roughly estimate (neglecting drag forces and hydrodynamic interactions) the time of travel from the equation of motion, $\triangle x \approx a * \Delta t^{2}$, which results 12 msecs, three orders of magnitude faster than disassembly purely by Brownian motion, as explained above. Particle tracking of experimental data provided us an average speed of 5-9 $\mu \mathrm{m} / \mathrm{s}$ for colloids switching between the DEP and MAG minima (depending on the electric field strength between $\mathrm{E}=0.13-0.25 \mathrm{~V} / \mu \mathrm{m}$, see Supplementary Information Table S1). Plugging this number to the drag formula gives an estimate of $0.5-0.9 \mathrm{pN}$ for drag forces applying to a $5 \mu \mathrm{m}$ sized colloid in DMSO. In addition, by using the magnetophoretic force formula we estimated the magnetic forces to be on the order of $1-5 \mathrm{pN}$ for the $5 \mu \mathrm{m}$ sized particles with a magnetic susceptibility $\chi=0.17$ and assuming that our $16 \mathrm{mT}$ permanent magnet applies a $2-3 \mathrm{mT}$ field to the chip $1 \mathrm{~mm}$ away from the magnet surface, see Supplementary Information for more description on calculations. Furthermore, the switching response time can be controlled by varying the applied electric field strength. The experimental response time with DEP switching changes linearly as a function of applied field, as expected (see Figure 3d). The combination of electric and magnetic field strength, particle dielectric and magnetic properties, and electrode geometry offer several adjustable parameters to tune the switching response as desired.

This approach can also be applied to reversibly assemble binary particle suspensions. We observed a hierarchical organization of the binary-sized colloidal particles, where larger-sized particles were 

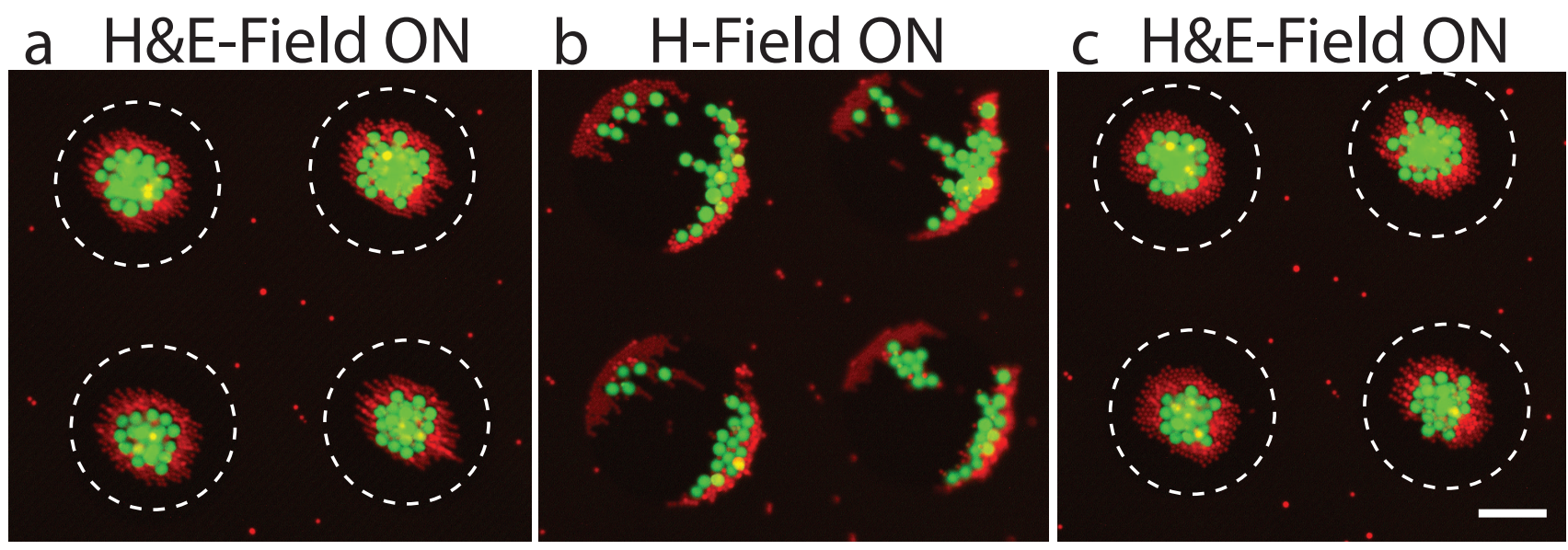

Figure 4: Hierarchical assembly of binary sized colloidal superparamagnetic particles by electric field and the switching of the hierarchical assembly by turning the electric field on and off while keeping the magnetic field on. Dashed circles outline the voids of the microfabricated nickel electrode. Panels of the image shows the field switching over time, namely at a) $0 \mathrm{~s}$, b) $75 \mathrm{~s}$ and c) $95 \mathrm{~s}$. Scale bar 40 $\mu \mathrm{m}$.

pushed to the core of the assembly and the smaller particles were surrounding the assembly when an electric field of $0.12 \mathrm{~V} / \mu \mathrm{m}$ strength was applied (Figure 4a and Figure S4). This can be attributed to fact that the larger particles experience a stronger force (dielectrophoretic force scales with the volume of the particle, $F_{\mathrm{DEP}} \sim r^{3}$ ) thereby faster response time with respect to the smaller ones. When the electric field was turned off, the magnetic field dissambles the superparamagnetic colloid assembly (Figure 4b). Here, we observed that $2.85 \mu \mathrm{m}$ sized (red) colloids (density $1.23 \mathrm{~g} / \mathrm{cm}^{3}$ ) were more magnetically responsive in comparison to the $7.9 \mu \mathrm{m}$ sized (green) colloids (density $1.1 \mathrm{~g} / \mathrm{cm}^{3}$, Bangs Laboratories, Inc., UMC4F), which is a direct outcome of their iron oxide content reflected in their densities.

The intriguing observation in Figure 4, that larger $7.9 \mu \mathrm{m}$ sized colloids respond to an external magnetic field faster than $2.85 \mu \mathrm{m}$ sized magnetic colloids is based on their magnetic contents of the colloids, which is $5.75 \mathrm{vol} \%$ for the smaller particles but is and $2.5 \mathrm{vol} \%$ for the $7.9 \mu \mathrm{m}$ sized colloids. The magnetic contents were estimated simply by using rule of mixtures and the final densities of the particles and the density of iron oxide $\left(\mathrm{Fe}_{3} \mathrm{O}_{4}\right)$ as $5 \mathrm{~g} / \mathrm{cm}^{3}$. The iron oxide content significantly alters the magnetic susceptibility of the particles, which was as low as $\chi_{m}=0.05^{41}$ for $7.9 \mu \mathrm{m}$ colloids and $\chi_{m}=0.39$ for the $2.85 \mu \mathrm{m}$ sized colloids. Thus, smaller colloids react faster to magnetic field and 
assemble at the edges of the nickel grid before the larger ones (see Figure 4b, see also Supplementary Information). As the electric field is turned on again, the assembly reverses to the initial state as a result of the state of external field potentials (see Figure 4c). In addition to creating hierarchical structures using different particle sizes, differences in particle susceptibility and/or dielectric properties can also be exploited. For example, combining magnetic and nonmagentic particles allows for the selective organization of those particles at different locations of the grid by tuning the electric field strength. We further showed the behaviour of a binary colloid system composed of magnetic and non-magnetic particles, which allows for selectively organize non-magnetic or magnetic colloids at difeent locations of the grid via adjusting the electric field strength (see Figure S5 for details).

We designed and microfabricated chips with $\mathrm{Ni}$ and $\mathrm{Au}$ patch geometries that allow spatial control of colloids simultaneously by magnetophoresis and dielectrophoresis. For superparamagnetic particles, this device offers a bistable potential landscape that can be navigated by turning on and off the electric and magnetic fields. Designing the potential minima for DEP in the vicinity of MAG potential minima allows to shuttle the colloids reversibly between the two potential minima. By applying different fields, we showed that colloids can reversibly switch between these two minima. We also demonstrated that this design can be used as an optical switch with response times comparable to the state of art and enables easy downscaling of the device for miniaturization goals, due to micron-size of the colloidal particles, which is used as the shutter. This assembly paradigm can also be applied to heterogeneous particle populations to construct dynamic, switchable, hierarchical assemblies, as demonstrated for a binary sized mixture of superparamagnetic colloids. The ability to shuttle between the two potential minima is a simple optical switch but we believe the tunability and flexibility provided by the additional control parameters by combining electric and magnetic fields can potentially be applied to more exotic applications, such as optical displays. 


\section{Methods}

Confocal Microscopy: We studied the samples using a Zeiss LSM 880 upright confocal scanning laser microscopy with a $20 \times 0.8$ NA Plan-Apochromat objective. For binary particle systems we used a fluorescence microscope of Leica with an inverted DM6000 stage.

Colloids: The spherical superparamagnetic particles of $4.69 \mu \mathrm{m}$ size (microparticles GmbH, PSMAG-RhB-S2538, $\chi_{m}=0.17$ ) and $2.85 \mu \mathrm{m}$ size (Bangs Laboratories, Inc., UMC3F, $\chi_{m}=0.39^{23}$ ), $7.9 \mu \mathrm{m}$ size (Bangs Laboratories, Inc., UMC4F, $\chi_{m}=0.05^{41}$ ) were commercially available and were used by dispersing in the mentioned solvents.

Electrode and sample fabrication: Gold grids were fabricated by standard photolithography. This involved dissolution of the exposed photoresist regions, subsequent e-beam evaporation of $4 \mathrm{~nm}$ titanium and $40 \mathrm{~nm}$ gold layers, and lift-off of the masking photoresist by sonication. On top of the gold electrodes another round of photolithography process was performed to plate nickel patches. For nickel we deposited $10 \mathrm{~nm}$ titanium followed by a $200 \mathrm{~nm}$ nickel layer. The sample cells were fabricated by sandwiching a micro-fabricated electrode with a counter plane indium tin oxide (ITO) electrode while the conductive faces looked into each other separated by spacer \#0 (0,09 mm - 0,12 $\mathrm{mm})$ or \#00 (0,06 mm - 0,08 mm) cover slips.

Finite element analyses: Finite element analyses were conducted by using Comsol 5.2 to estimate the electric and magnetic field strength landscape of the micropatterned electrodes imposed to the particles. For magnetic field calculations we used relative magnetic permeabilities of air $\left(\mu_{r}(\right.$ air $\left.)=1\right)$ and of nickel $\left(\mu_{r}(\right.$ nickel $\left.)=200\right)$, and magnetization of the permanent magnet as 105,000 A/m. Furthermore, for electric fields we used a field strength of $0.1 \mathrm{~V} / \mu \mathrm{m}$ applied between two electrodes separated with a distance of $100 \mu \mathrm{m}$. Despite being semi-quantitative and not including frequency response, our simulations reliably provided how the field strength changed in 3D for a given potential between the micro-patterned and unpatterned electrodes.

\section{Supporting Information}

The Supporting Information is available free of charge on the ACS Publications website. Figures 
showing the calculations of the field strengths for a micropatterened grid and assembly behaviour of binary colloid system and a description on calcualtions are provided.

\section{Acknowledgements}

Prof. Andre Studart (ETH, Complex Materials ), Prof. Jan Vermant (ETH, Soft Materials), Prof. Eric Dufresne (ETH, Living Materials) and Prof. Askin Kocabas (Koc University, Physics) are acknowledged for fruitful discussions and support. A.F.D. acknowledges the Swiss National Science Foundation (Ambizione grant, number PZ00P2_148040) for financial support and ETH cleanroom, FIRST, for microfabrication facilities and ETH microscopy center Scopem for imaging. H.R.V. was

partially supported by a Marie Skodowska-Curie Intra European Fellowship (G.A. No. 708349SPCOLPS) within Horizon 2020.

\section{References}

(1) Grzelczak, M.; Vermant, J.; Furst, E. M.; Liz-Marzan, L. M. Directed Self-Assembly of Nanoparticles. ACS Nano 2010, 4, 3591-3605.

(2) Yethiraj, A.; Thijssen, J. H. J.; Wouterse, A.; Blaaderen, A. v. Large-Area Electric-FieldInduced Colloidal Single Crystals for Photonic Applications . Adv Mater 2004, 16, 596-600.

(3) Xia, Y.; Gates, B.; Li, Z. Y. Self-Assembly Approaches to Three-Dimensional Photonic Crystals . Adv Mater 2001, 13, 409-413.

(4) Beltramo, P. J.; Schneider, D.; Fytas, G.; Furst, E. M. Anisotropic Hypersonic Phonon Propagation in Films of Aligned Ellipsoids. Phys. Rev. Lett. 2014, 113, 205503.

(5) Cheng, W.; Wang, J.; Jonas, U.; Fytas, G.; Stefanou, N. Observation and Tuning of Hypersonic Bandgaps in Colloidal Crystals . Nature Materials 2006, 5, 830-836.

(6) Talapin, D. V.; Lee, J.-S.; Kovalenko, M. V.; Shevchenko, E. V. Prospects of Colloidal Nanocrystals for Electronic and Optoelectronic Applications. Chem Rev 2010, 110, 389-458. 
(7) Holtz, J. H.; Asher, S. A. Polymerized Colloidal Crystal Hydrogel Films as Intelligent Chemical Sensing Materials . Nature 1997, 389, 829-832.

(8) Shipway, A. N.; Katz, E.; Willner, I. Nanoparticle Arrays on Surfaces for Electronic, Optical, and Sensor Applications. ChemPhysChem 2000, 1, 18-52.

(9) Yablonovitch, E.; Gmitter, T. J. Photonic Band Structure- the Face-Centered-Cubic Case . Phys. Rev. Lett. 1989, 63, 1950-1953.

(10) Yablonovitch, E. Inhibited Spontaneous Emission in Solid State Physics and Electronics . Phys. Rev. Lett. 1987, 58, 2059-2062.

(11) Blaaderen, A. v.; Ruel, R.; Wiltzius, P. Template-Directed Colloidal Crystallization . Nature 1997, 385, 321-324.

(12) Dong, A.; Chen, J.; Vora, P. M.; Kikkawa, J. M.; Murray, C. B. Binary Nanocrystal Superlattice Membranes Self-Assembled at the Liquid-Air Interface . Nature 2010, 466, 474-477.

(13) Velikov, K. P.; Moroz, A.; Blaaderen, A. v. Photonic Crystals of Core-Shell Colloidal Particles . Appl. Phys. Lett. 2002, 80, 49.

(14) Mikhael, J.; Roth, J.; Helden, L.; Bechinger, C. Archimedean-Like Tiling on Decagonal Quasicrystalline Surfaces . Nature 2008, 454, 501-504.

(15) Yin, Y.; Lu, Y.; Gates, B.; Xia, Y. Template-Assisted Self-Assembly: a Practical Route to Complex Aggregates of Monodispersed Colloids with Well-Defined Sizes, Shapes, and Structures . J Am Chem Soc 2011, 123, 8718-8729.

(16) Aizenberg, J.; Braun, P. V.; Wiltzius, P. Patterned Colloidal Deposition Controlled by Electrostatic and Capillary Forces . Phys. Rev. Lett. 2000, 84, 2997-3000.

(17) Hayward, R. C.; Saville, D. A.; Aksay, I. A. Electrophoretic Assembly of Colloidal Crystals with Optically Tunable Micropatterns . Nature 2000, 404, 56-59. 
(18) Korkut, S.; Saville, D. A.; Aksay, I. A. Collodial Cluster Arrays by Electrohydrodynamic Printing . Langmuir 2008, 24, 12196-12201.

(19) Lu, Y.; Yin, Y.; Xia, Y. A Self-Assembly Approach to the Fabrication of Patterned, TwoDimensional Arrays of Microlenses of Organic Polymers. Adv Mater 2001, 13, 34-37.

(20) Kralchevsky, P. A.; Denkov, N. D.; Paunov, V. N.; Velev, O. D.; Ivanov, I. B.; Yoshimura, H.; Nagayama, K. Formation of 2-Dimensional Colloid Crystals in Liquid-Films Under the Action of Capillary Forces. J Phys-Condens Mat 1994, 6, A395-A402.

(21) Yellen, B. B.; Hovorka, O.; Friedman, G. Arranging Matter by Magnetic Nanoparticle Assemblers. Proc. Natl Acad. Sci. USA 2005, 102, 8860-8864.

(22) Kauffmann, P.; Ith, A.; O’Brien, D.; Gaude, V.; Boué, F.; Combe, S.; Bruckert, F.; Schaack, B.; Dempsey, N. M.; Haguet, V.; Reyne, G. Diamagnetically Trapped Arrays of Living Cells Above Micromagnets . Lab Chip 2011, 11, 3153-3161.

(23) Demirörs, A. F.; Pillai, P. P.; Kowalczyk, B.; Grzybowski, B. A. Colloidal Assembly Directed by Virtual Magnetic Moulds . Nature 2013, 503, 99-103.

(24) Dong, A.; Ye, X.; Chen, J.; Murray, C. B. Two-Dimensional Binary and Ternary Nanocrystal Superlattices: the Case of Monolayers and Bilayers . Nano Lett 2011, 11, 1804-1809.

(25) Rossi, L.; Sacanna, S.; Irvine, W. T. M.; Chaikin, P. M.; Pine, D. J.; Philipse, A. P. Cubic Crystals From Cubic Colloids . Soft Matter 2011, 7, 4139-4142.

(26) Chen, Q.; Bae, S. C.; Granick, S. Directed Self-Assembly of a Colloidal Kagome Lattice . Nature 2011, 469, 381-384.

(27) Velikov, K. P.; Christova, C. G.; Dullens, R.; Blaaderen, A. v. Layer-by-Layer Growth of Binary Colloidal Crystals . Science 2002, 296, 106-109.

(28) Hoogenboom, J. P.; Rétif, C.; de Bres, E.; van de Boer, M.; van Langen-Suurling, A. K.; Romijn, J.; Blaaderen, A. v. Template-Induced Growth of Close-Packed and Non-Close-Packed Colloidal Crystals During Solvent Evaporation . Nano Lett 2004, 4, 205-208. 
(29) Jiang, P.; Prasad, T.; McFarland, M. J.; Colvin, V. L. Two-Dimensional Non-Close-Packed Colloidal Crystals Formed by Spincoating . Appl. Phys. Lett. 2006, 89, 011908.

(30) Xia, Y. N.; Yin, Y. D.; Lu, Y.; McLellan, J. Template-Assisted Self-Assembly of Spherical Colloids Into Complex and Controllable Structures . Adv. Funct. Mater. 2003, 13, 907-918.

(31) Zhang, Q.; Janner, M.; He, L.; Wang, M.; Hu, Y.; Lu, Y.; Yin, Y. Photonic Labyrinths: Two-Dimensional Dynamic Magnetic Assembly and in Situ Solidification. Nano Lett 2013, 13 , $1770-1775$.

(32) He, L.; Wang, M.; Zhang, Q.; Lu, Y.; Yin, Y. Magnetic Assembly and Patterning of General Nanoscale Materials through Nonmagnetic Templates. Nano Lett 2013, 13, 264-271.

(33) Bartlett, A. P.; Agarwal, A. K.; Yethiraj, A. Dynamic Templating of Colloidal Patterns in Three Dimensions with Nonuniform Electric Fields. Langmuir 2011, 27, 4313-4318.

(34) Whitehead, J. B.; Zumer, S.; Doane, J. W. Light Scattering From a Dispersion of Aligned Nematic Droplets . Journal of Applied Physics 1993, 73, 1057-1065.

(35) Ren, H.; Wu, S.-T. Optical Switch Using a Deformable Liquid Droplet . Optics Letters 2010, 35, 3826-3828.

(36) Yang, D. K.; Chien, L. C.; Doane, J. W. Cholesteric Liquid Crystal/Polymer Dispersion for Haze-Free Light Shutters. Appl. Phys. Lett. 1992, 60, 3102-3104.

(37) Hayes, R. A.; Feenstra, B. J. Video-Speed Electronic Paper Based on Electrowetting . Nature 2003, 425, 383-385.

(38) Pohl, H. A. Dielectrophoresis: The Behavior of Neutral Matter in Nonuniform Electric Fields; Cambridge University Press: Cambridge ; New York, 1978.

(39) Crassous, J. J.; Demirörs, A. F. Multiscale Directed Self-Assembly of Composite Microgels in Complex Electric Fields . 2016, 13, 88-100.

(40) Rosensweig, R. E. Ferrohydrodynamics; Courier Dover Publications, 1997. 
(41) Chen, A.; Sooryakumar, R. Patterned Time-Orbiting Potentials for the Confinement and Assembly of Magnetic Dipoles . Scientific Reports 2013, 3, 3124. 


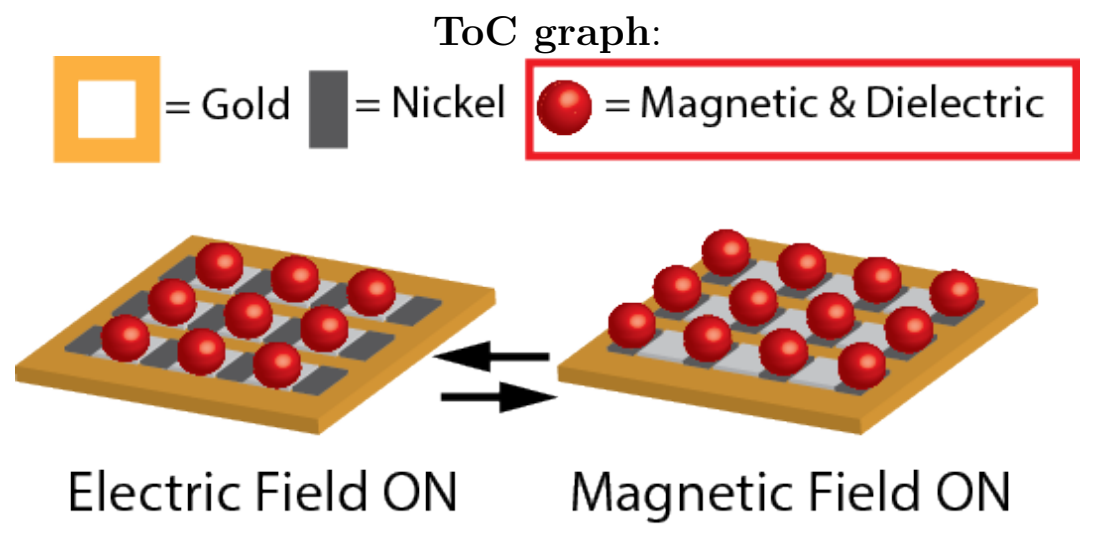

